une raison pour nous encourager à croire que l'infection d'un quartier provoque un certain degré d'immunité dans les autres.

On doit aussi remarquer que, dans les essais de vaccination, il n'a pas pu encore être fixé de règles indiquant la quantité de germes nécessaires à introduire chez une vache, par voie sous-cutanée, pour obtenir un degré suffisant d'immunisation. La quantité de vaccin à injecter est bien différente chez les divers expérimentateurs. SemlemanN, par exemple, qui dans ses derniers travaux croit pouvoir tirer la conclusion que la vaccination est sans effet, a utilisé, dans ses expériences, des doses de vaccin de 5 à $20 \mathrm{~cm}^{3}$ d'une dilution de cultures en tubes d'agar, de $5 \mathrm{~cm}^{3}$ par tube, sans donner le nombre de germes par centimètre cube de son vaccin. Nos expériences nous ont montré que des injections de $300 \mathrm{~cm}^{3}$ de cultures vivantes, obtenues dans du bouillon avec sérum, foie, cœur et lactose, qui produit un nombre de germes bien plus grand que l'agar, n'ont donné aucune réaction perceptible sur les vaches chez qui nous les avons faites. En vaccinothérapie, nous nous approchons de la dose préventive et curative, quand celle-ci provoque les premières réactions thermiques ou d'augmentation de l'indice opsonique ou de réactions leucocytaires et autres.

Jamais on n'a pu fixer la dose de vaccin à donner à une vache pour la mammite, ni l'intervalle entre les doses. Bien que nous n'ayons pas eu l'occasion d'étudier ce point, comme nous désirions le faire, nous sommes bien loin de croire que les doses de 5 à $20 \mathrm{~cm}^{3}$ de suspensions de cultures d'agar, à intervalle de deux ou trois semaines entre chaque injection, comme SEELEMAN l'a fait, soient suffisantes ou se rapprochent de la dose nécessaire.

(A suivre).

\title{
L'IMPORTANCE DU CUIVRE COMME MATÉRIAU POUR LA CONSTRUCTION DES APPAREILS EMPLOYÉS EN LAITERIE
}

par

le Dr A. KARSTEN

Au cours de ces dernières années, il a été fait de grands "efforts pour remplacer le cuivre dans la construction des appareils électriques par d'autres métaux ou certains alliages, comme par exemple l'aluminium, certaines qualités d'aciers spéciaux, mais il semble que ces efforts n'aient pas donné de grands résultats.

Il y a quelques années, Mederer, de Munich, avait d'ailleurs publié une étude intéressante intitulée "Le cuivre en laiterie », dans laquelle cet Auteur souligne à juste titre que le cuivre reste toujours 
le matériau le meilleur pour la construction des appareils comme les pasteurisateurs, les réfrigérants, ainsi que les tuyauteries utilisées dans les laiteries.

On peut dire que jusqu'au milieu du siècle dernier la construction des appareils employés en laiterie est restée entre les mains des chaudronniers qui disposaient d'une très longue pratique de la question, puis le travail du cuivre a été ensuite entrepris par des établissements industriels bénéficiant de moyens beaucoup plus importants et il en est résulté des perfectionnements nombreux apportés au traitement du cuivre, grâce à de nombreuses recherches métallurgiques sur ce métal.

On sait qu'en Allemagne, des règlements très sévères s'appliquent à toutes les opérations que peut subir le lait et en particulier, il est dit dans le premier décret de la loi du Reich du 15 mai 1931, que toutes les parties d'appareils ou de conduites pouvant être au contact du lait, sauf les appareils entièrement ouverts, d'un nettoyage facile, doivent être étamées. Or, l'étamage électrolytique, pas plus que l'étamage à chaud, n'offrent de difficulté, depuis surtout que les recherches remarquables de M. CxмвоLIste présentées par l'Auteur à la séance du 22 mai 1935 de la Société de chimie industrielle ont permis de porter ces technqiues à un très haut degré de perfection. C'est ainsi qu'aujourd'hui il est possible de réaliser sur le cuivre un étamage impeccable, d'une épaisseur uniforme et avant tout ne présentant pas de pores. C'est évidemment ce revêtement sans défaut, absolument exempt de trous, qui correspond aux exigences des laiteries, puisqu'il exclut avec certitude la formation de couples locaux susceptibles de provoquer une corrosion du métal.

C'est un vieux fait d'expérience que les matériaux métalliques ont une résistance à la corrosion d'autant plus élevée qu'ils sont plus purs. C'est pour tenir compte de cette observation que la "United States Metals Refining Company" dans son usine de cuivre de Carteret a mis au point, depuis déjà plusieurs années, un procédé de raffinage connu sous le nom de procédé " $O$. F. H. C. ». Ce procédé permet d'obtenir une désoxydation complète du métal sans que pour cela la teneur en phosphore restant dans le produit soit supérieure à 0,015 à $0,025 \%$. Le métal de grande pureté acquiert de ce fait une conductibilité électrique particulièrement élevée.

Le procédé O. F. H. C. consiste à faire couler le mótal que l'on a liquéfié au travers d'une couche épaisse de charbon de bois pur qui élimine les dernières traces d'oxygène contenues dans le cuivre. Après cette désoxydation, le métal coule par l'intermédiaire d'une conduite de construction spéciale dans des récipients oì il se trouve parfaitement protégé contre l'oxydation par une atmosphère de gaz protecteur. Ce gaz, fourni par un générateur à charbon de bois, 
a la composition suivante : 27 à $28 \%$ d'oxyde de carbone, $0,5 \%$ d'acide carbonique, le reste d'azote. Il est entièrement exempt d'hydrocarbures, de vapeur d'eau et d'autres constituants nuisibles. Le métal purifié par ce procédé, et en particulier exempt d'oxyde cuivreux, constitue un matériau excellent pour la construction de tous les appareils et conduites utilisés en laiterie.

On utilise également depuis plusieurs années aux Etats-Unis des alliages de cuivre qui se caractérisent par des propriétés mécaniques particulièrement élevées et qui sont principalement destinés à la fabrication de récipients devant résister à la pression. L'emplui de ces alliages permet d'utiliser des épaisseurs plus faibles de métal et facilitent par conséquent la soudure. Ces alliages conviennent particulièrement aux appareils dans lesquels on chauffe l'eau nécessaire aux services de lavage et de nettoyage dans les laiteries. Avec la collaboration de la Verein Deutsches Kupfer Institute, le Dr M. Mengeringhausen a examiné d'une façon très approfondie les caractéristiques de tôles fabriquées avec ces alliages qui contiennent certains constituants destinés à le durcir, notamment du silicium. Les résultats qu'il a obtenus sont résumés dans le tableau ci-dessous :

No Désignation

1. E-CU

DIN VDE 500 .

2. Matériau 240 .

3. Sicufal . .

4. Matériau 132 .

5. Mandura . .
Résistance à la rupture

(Doux)

$21 \mathrm{~kg} / \mathrm{mm}^{2}$
$40 \mathrm{~kg} / \mathrm{mm}^{2}$
$50 \mathrm{~kg} / \mathrm{mm}^{2}$
$33 / 35 \mathrm{~kg} / \mathrm{mm}^{2}$
$40 / 42 \mathrm{~kg} / \mathrm{mm}^{2}$

$21 \mathrm{~kg} / \mathrm{mm}^{2}$

$30 \mathrm{~kg} / \mathrm{mm}^{2}$

$55 \mathrm{~kg} / \mathrm{mm}^{2}$

$48 / 50$ (trempé et demi-trempé)

56/60 (trempé et demi-trempé)
Allongement en $\%$

(Doux) (Trempé)

$\begin{array}{lc}35 & 4 \\ 50 & 20 \\ 40 & \\ 32 / 40 & \\ 25 / 22 & 6 / 5 \\ & \\ & \\ & \\ & \end{array}$

A ce groupe des alliages de cuivre de haute résistance mécanique il faut joindre également d'autres alliages qui se comportent d'une façon analogue, comme par exemple le Kuprodur. Par suite de leur résistance mécanique augmentée, il est possible de diminuer l'épaisseur des parois des appareils construits avec ces métaux et il en résulte une économie de matière première. Si du fait de la diminution de l'épaisseur des parois, lạ rigidité devient insuffisante, on peut facilement éviter des déformations éventuelles en renforçant les appareils par des armatures, des supports, des cercles, etc. Notons enfin que la présence de faibles proportions de inanganèse et de silicium dans le cuivre augmente notablement la résistance à l'usure du métal.

En ce qui concerne la fabrication des tuyaux de cuivre de haute qualité, cette opération a été énormément favorisée par le procédé 
dit de coulée centrifuge. Pratiquement, le cuivre liquide est coulé dans des conditions parfaitement déterminées dans des moules en acier tournant rapidemont. Le cuivre se dépose à la périphérie du moule et, sous l'influence des forces centrifuges, on constate que son grain est nettement amélioré et sa densité augmentée. En outre, toutes les impuretés plus légères, comme par exemple les gaz, les inclusions de scories, etc., sont chassées, de sorte qu'on obtient des pièces parfaitement homogènes et exemptes de pores. Par ce procédé de coulée centrifuge, on a pu obtenir des tuyaux ayant jusqu'à 10 mètres de longueur et de section et d'épaisseur très variable.

Le développement des techniques modernes de soudure a permis également d'améliorer les méthodes de travail du cuivre. La mise au point de machines à guidage automatique qui travaillent à grande vitesse et avec une grande sécurité de marche a permis de réaliser la soudure impecable du cuivre pur. Les soudures obtenues de cette façon ne contiennent aucun constituant étranger, elles sont solides et résistent à la corrosion. Les progrès réalisés dans la soudure moderne ont permis également de surmonter toutes les diffieultés qui rendaient jadis difficile le travail des différents alliages de cuivre.

En ce qui concerne l'avenir, il semble 'qu'un alliage découvert récemment se répandra de plus en plus dans la construction des appareils de laiterie; il s'agit de l'alliage dénommé Sonder-bronze DKS qui est exempt de zinc et d'étain et qui, tout en ayant des propriétés mécaniques excellentes, se caractérise également par une grande résistance à la corrosion. Cela tient à la forme cristalline particulière de cet alliage cuivre-silicium. Pour avoir plus de détails sur ses qualités, on consultera les Demag-Nachrichten, $11^{\mathrm{e}}$ année, B. $\mathrm{n}^{\circ} 1,1937$, p. 5 .

Le travail des tuyauteries en cuivre peut être aujourd'hui effectué par tout installateur quelque peu exercé, sans qu'il se produise des difficultés particulières. Le métal peut être facilement soudé au chalumeau ou à la soudure forte, contrairement au cas des conduites en fer. D'autre part, il est très facile de courber les tuyaux, de sorte que l'on peut faire des branchements sans être dans l'obligation de multiplier le nombre des raccords.

Les tubes de cuivre dont le diamètre est inférieur à $32 \times 35$ millimètres peuvent être pliés à froid, même selon un rayon très faible et sans qu'il soit nécessaire de les remplir de résine, en disposant à cet effet d'un outillage très simple. Si le tuyau a un diamètre de 15 millimètres, on peut le courber en quelques secondes seulement.

Les tubes dont le diamètre dépasse 35 millimètres extérieurement doivent être remplis avec soin de sable fin sec. On les chauffe ensuite dans un peu de charbon de bois bien rouge, on les courbe, puis on les refroidit rapidement, en les arrosant d'eau froide. 
Pour l'exécution des raccords, on a imaginé des modes de raccordement très simples, par exemple les raccords à pince "Rapid " ou "Instantor ", les raccords à filet ou enfin le raccord avec soudure "Bänninger ».

Par suite de sa résistance de rupture élevée, de son élasticité et de son excellente résistance aux vibrations, le tube de cuivre est de beaucoup supérieur à tous les autres tubes lorsqu'il s'agit d'installations de conduites d'eau. En effet, il est très difficilement endom. mageable, soit sous l'influence de coups de bélier, soit par suite de la congélation de l'eau. L'intérieur d'une conduite d'eau en cuivre se recouvre rapidement d'une couche protectrice excessivement mince, qui reste par conséquent lisse et qui s'oppose à toute incrustation de sels calcaires. L'expérience a montré que la durée d'emploi d'un tube de cuivre, poli intérieurement, s'élève à au moins 50 ans. Le tube de cuivre est également à recommander par suite de sa résistance et de son bon marché pour l'installation des conduites parcourues par des courants d'eau chaude ou des courants d'eau froide. Il ne faut pas oublier que les récipients et les conduites en cuivre ont une action bactéricide (oligodynamique) importante qui n'est que très légèrement inférieure à celle des ustensiles d'argent.

On peut dire que le degré de perfection atteint aujourd'hui par la construction des appareils employés en laiterie aurait difficilement pu être obtenu, sans l'emploi du cuivre comme matériau. Malheureusement aujourd'hui, on tend à oublier ce point et surtout on n'apporte pas assez d'attention à l'entretien des appareils. Le cuivre poli ne peut être nettoyé qu'avec des agents de nettoyage fortement dispersés et exempts d'impuretés telles que grains de sable, fragments métalliques ou de verre. Si les appareils sont parfaitement entretenus, l'étamage devient superflu et même parfois indésirable, car il supprime l'action oligodynamique du cuivre. Les surfaces de euivre qui ne sont pas au contact des produits laitiers seront protégées contre le ternissement par application d'un vernis transparent convenable. Pour obtenir des effets décoratifs, "on peut également faire appel à différents procédés de la Verein Deutsehes Kupfer Institut " grâce auxquels on peut communiquer au cuivre une coloration superficielle très plaisante et qui garantit en outre le métal, d'une façon efficace, contre la formation d'oxydes dans les salles chaudes et humides.

En résumé, on peut prévoir que le matériau cuivre ne pourra pas être évincé dans la construction des appareils et des installations de laiterie, sans qu'il en résulte des inconvénients parfois très sérieux. 